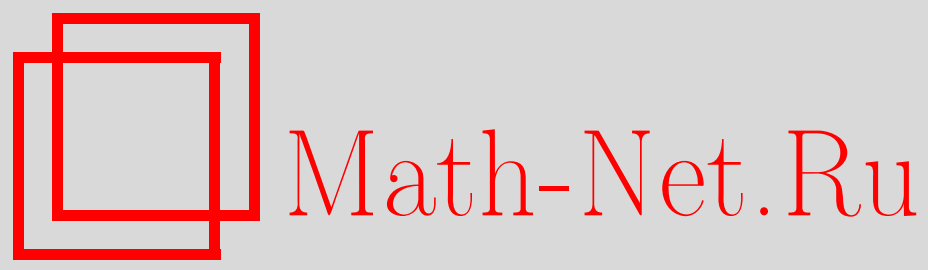

А. М. Гомилко, K теории $J$-самосопряженных возмущений самосопряженных операторов, Функи. анализ и его прил., 1996, том 30, выпуск 1, 61-63

DOI: https://doi.org/10.4213/faa506

Использование Общероссийского математического портала MathNet.Ru подразумевает, что вы прочитали и согласны с пользовательским соглашением

http://www . mathnet.ru/rus/agreement

Параметры загрузки:

IP: 54.197 .217 .227

26 апреля 2023 г., 05:28:09

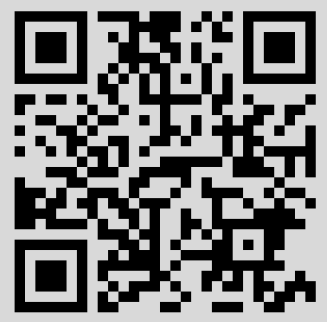


УдК 513.8

\title{
K теории $J$-самосопряженных возмущений самосопряженных операторов
}

\author{
(c) 1996. А. М. Гомилко
}

Пусть $H$ - бесконечномерное сепарабельное гильбертово пространство со скалярным произведением $(\cdot, \cdot)$ и единичным оператором $I$. Введем в рассмотрение пространство Крейна $\mathscr{H}=H \oplus H$ (см. [1]) со скалярным произведением $(\cdot, \cdot)_{\mathscr{H}}$ и индефинитным скалярным произведением $\langle\cdot, \cdot\rangle=(J \cdot, \cdot)_{\mathscr{H}}$, где оператор $J$ определен формулой $J=\left(\begin{array}{cc}I & 0 \\ 0 & -I\end{array}\right)$. Пусть $A_{1}, A_{2}$ - вполне непрерывные самосопряженные линейные операторы в пространстве $H$, а $\mathscr{K}$ - конечномерный $J$-самосопряженный оператор в $\mathscr{H}$, т. е. $\operatorname{dim} \mathscr{K} \mathscr{H}<\infty$ и $J \mathscr{K}=\mathscr{K}^{*} J$. Введем в рассмотрение семейство вполне непрерывных $J$-самосопряженных операторов

$$
\mathscr{A}(\varepsilon)=\mathscr{A}+\varepsilon \mathscr{K}, \quad \mathscr{A}=\left(\begin{array}{cc}
A_{1} & 0 \\
0 & A_{2}
\end{array}\right), \quad \varepsilon \geqslant 0 .
$$

При $\varepsilon=0$ оператор $\mathscr{A}=\mathscr{A}(0)$ является самосопряженным и поэтому имеет чисто вещественный спектр. С другой стороны, если операторы $A_{1}, A_{2}$ из (1) не имеют общих собственных значений, то для любого ненулевого собственного значения $\lambda_{0}$ оператора $\mathscr{A}$ соответствующее собственное подпространство $\mathscr{L}_{\lambda_{0}}(\mathscr{A})$ является равномерно $J$-дефинитным:

$$
|\langle x, x\rangle| \geqslant c\|x\|_{\mathscr{H}}^{2} \quad \forall x \in \mathscr{L}_{\lambda_{0}}(\mathscr{A}), c>0 .
$$

Тогда $[2,3]$ найдутся такие $\delta>0, \varepsilon_{0}>0$, что для любого $\varepsilon \in\left[0, \varepsilon_{0}\right)$ спектр оператора $\mathscr{A}(\varepsilon)$ в окрестности $\left|\lambda-\lambda_{0}\right|<\delta$ является вешественным и имеет суммарную алгебраическую кратность, равную размерности пространства $\mathscr{L}_{\lambda_{0}}(\mathscr{A})$. В связи с этим обстоятельством возникает вопрос: верно ли, что для фиксированной пары операторов $\mathscr{A}, \mathscr{K}$ с указанными выше свойствами найдется такое достаточно малое $\varepsilon_{0}>0$, что при $\varepsilon \in\left[0, \varepsilon_{0}\right)$ оператор $\mathscr{A}(\varepsilon)$ из $(1)$ имеет не более конечного числа невещественных собственных значений? (Отметим, что близкие вопросы возникают в механике сплошной среды.) В данной заметке показано, что в общем случае ответ на поставленный вопрос является отрицательным даже при одномерном возмущении оператора $\mathscr{A}$ и выполнении условия подчинения

$$
\left\|\mathscr{A}^{-1} \mathscr{K}\right\|_{\mathscr{H}}<\infty .
$$

Для построения соответствующего примера введем необходимые определения. Пусть $A$ - положительный вполне непрерывный оператор в $H$ с простым спектром и $\left\{e_{k}\right\}_{k=1}^{\infty}$ - ортогональный базис в $H$, составленный из собственных векторов оператора $A$. Пусть $f \in H$ - произвольный фиксированный вектор, удовлетворяющий условию

$$
\left(f, e_{k}\right) \neq 0, \quad k=1,2, \ldots
$$


Введем в рассмотрение в пространстве $H$ самосопряженные операторы $T$, $A_{1}, A_{2}$ :

$$
T x_{1}=\left(x_{1}, f\right) f, \quad x_{1} \in H, \quad A_{1}=(I+T)^{-1 / 2} A(I+T)^{-1 / 2}, \quad A_{2}=A .
$$

Определим одномерный $J$-самосопряженный в $\mathscr{H}$ оператор формулой

$$
\mathscr{K}=\left(\begin{array}{cc}
T(I+T)^{-1} & -T(I+T)^{-1 / 2} \\
T(I+T)^{-1 / 2} & -T
\end{array}\right) .
$$

Простота спектра положительного оператора $A$ и условие (4) на вектор $f$ обеспечивают справедливость следующего утверждения.

ПредЛОЖЕнИЕ. Операторы $A_{1}$ и $A_{2}$ из (5) не имеют общих собственных значений.

Таким образом, для любого собственного подпространства $\mathscr{L}_{\lambda_{0}}(\mathscr{A})$ оператора $\mathscr{A}$, определенного согласно формулам (1) и (5), выполнено условие дефинитности (2).

Теорема. При любом $\varepsilon>0$ оператор $\mathscr{A}(\varepsilon)$, определенный формулами (1), (5), (6), не имеет собственных значений на интервале $\lambda \in(0,4 \varepsilon)$.

ДокаЗАТЕЛЬСтво. Пусть $\varepsilon>0$ фиксировано и $\lambda$ - собственное значение оператора $\mathscr{A}(\varepsilon)$. Тогда, согласно определению оператора $\mathscr{A}(\varepsilon)$, найдутся такие векторы $y_{j} \in H, j=1,2$, не равные нулю одновременно, для которых справедливы равенства

$$
(A-\lambda T-\lambda I) y_{1}=-\varepsilon T\left(y_{1}-y_{2}\right), \quad(A-\lambda I) y_{2}=-\varepsilon T\left(y_{1}-y_{2}\right) .
$$

При этом в случае вещественности собственного значения $\lambda$ из (7) нетрудно получить равенство

$$
(2 \varepsilon-\lambda) \operatorname{Re}\left[\left(y_{1}, f\right)\left(f, y_{2}\right)\right]=\varepsilon\left(\left|\left(y_{1}, f\right)\right|^{2}+\left|\left(y_{2}, f\right)\right|^{2}\right) .
$$

С другой стороны, в силу условий (4) и (7) заключаем, что

$$
\left|\left(y_{1}, f\right)\right|+\left|\left(y_{2}, f\right)\right| \neq 0,
$$

а тогда из (8) получаем, что $\lambda$ не может принадлежать интервалу $(0,4 \varepsilon)$. Теорема доказана.

Используя теорему М. В. Келдыша [4, с. 314] о полноте системы корневых векторов и спектре слабо возмушенного самосопряженного оператора, из доказанной теоремы имеем следующее утверждение, дающее отрицательный ответ на поставленный вопрос о количестве невещественных собственных значений оператора $\mathscr{A}(\varepsilon)$.

СлЕДСТВИЕ. Пусть вполне непрерывный положительный оператор $A$ имеет простой спектр и конечныи порлдок, а вектор $f$, удовлетворяющий условию (4), имеет вид $f=A g, g \in H$. Тогда для любого $\varepsilon>0$ оператор $\mathscr{A}(\varepsilon)$, определенный согласно $(1),(5),(6)$, имеет бесконечное число невещественных собственных значений.

Отметим, что если для собственных пространств невозмущенного оператора $\mathscr{A}$ не требовать выполнения условия дефинитности (2), то возможна такая ситуация, когда для любого $\varepsilon>0$ все собственные значения возмущения $\mathscr{A}(\varepsilon)$ будут 
невещественными. Приведем соответствующий пример. Оператор

$$
\mathscr{A}(\varepsilon)=\left(\begin{array}{cc}
A & 0 \\
0 & A
\end{array}\right)+\varepsilon\left(\begin{array}{cc}
0 & -T \\
T & 0
\end{array}\right)
$$

унитарно эквивалентен оператору

$$
V \mathscr{A}(\varepsilon) V^{-1}=\left(\begin{array}{cc}
A+i \varepsilon T & 0 \\
0 & A-i \varepsilon T
\end{array}\right), \quad V=\frac{1}{\sqrt{2}}\left(\begin{array}{cc}
I & i I \\
i I & I
\end{array}\right) .
$$

При этом, если $T$ определен согласно (5) и выполнено условие (4), то все собственные значения операторов $A \pm i \varepsilon T$ для любого значения $\varepsilon>0$ являются невещественными, а значит, таким же свойством обладают и операторы $\mathscr{A}(\varepsilon)$, $\varepsilon>0$.

Автор благодарит Н. Д. Копачевского за полезные обсуждения.

\section{ЛитературА}

1. Азизов Т. Я., Иохвидов И. С. Основы теории линейных операторов в пространствах с индефинитной метрикой. Наука, М. (1986). 2. Дергузов В. И. Матем. сб., 63, вып. 4, 591-619 (1964). 3. Крейн $М . Г$. Введение в геометрию индефинитных $J$-пространств и теорию операторов в этих пространствах. Вторая летняя матем. школа, Т. 1, Наукова думка, Киев (1965), с. 15-92. 4. Гохберг И. Ц., Крейн М. Г. Введение в теорию линейных несамосопряженных операторов. Наука, М. (1965).

Институт гидромеханики

НАН Украины, Киев

Поступило в редакцию 15 июля 1994 г.

УдК 517.946

\section{Асимптотика усредненного лагранжиана для модели медленной диффузии с нелинейным вырождением}

$$
\text { (C) } 1996 . \quad \text { С. М. Козлов }
$$

В работе исследуется асимптотическое поведение усредненного лагранжиана при малом градиенте конщентрации для следующей модели нелинейной вырожденной диффузии. Предположим, что имеется локальная энергия вида

$$
l_{0}\left(\xi_{1}, \xi_{2}\right)=\frac{1}{2}\left(\left|\xi_{1}\right|-1\right)_{+}^{2}+\frac{1}{2}\left|\xi_{2}\right|^{2},
$$

где $(r)_{+}=\max \{r, 0\}$. С тем чтобы ввести зависимость от пространственной переменной, зададимся векторным полем $v(x), x \in \mathbb{R}^{2}$, таким, что $v(x) \neq 0$ почти везде. Определим векторное поле единичных векторов $\alpha(x)=v(x) /|v(x)|$ и обозначим через $T_{\alpha}$ матрицу поворота на угол $\alpha$ :

$$
T_{\alpha} \xi=\left(\begin{array}{cc}
\cos \alpha & \sin \alpha \\
-\sin \alpha & \cos \alpha
\end{array}\right)\left(\begin{array}{l}
\xi_{1} \\
\xi_{2}
\end{array}\right)
$$

Тогда по этим данным построим пространственно неоднородный лагранжиан по следующей формуле:

$$
L(x, \xi)=l_{0}\left(T_{\alpha(x)} \xi\right)
$$

